\title{
The Integrative Role of Magnetic Resonance Cholangiopancreatography and Percutaneous Transhepatic Cholangiography in Malignant Obstructive Jaundice
}

Hayam A. Abdellatif ${ }^{1}$, Omar A. Hamada ${ }^{2}$, Mohamed S. Elzawawi ${ }^{1}$, Mohamed A. Deif ${ }^{2}$

${ }^{1}$ Department of Radio-diagnosis, Faculty of Medicine, Menoufia University, Egypt.

${ }^{2}$ Department of Radiology, National Liver Institute, Menoufia University, Egypt.

*Correspondence author: Hayam Abdelmonsif Abdellatif, Mobile: (+20) 01098057324, Email: hayam_drar@yahoo.com

\begin{abstract}
Background: Magnetic resonance cholangiopancreatography (MRCP) and percutaneous trans-hepatic cholangiography (PTC) are established techniques for the evaluation of intra-hepatic and extra-hepatic bile ducts in patients with malignant hepato-biliary diseases. PTC has procedure-related complications. MRCP is accurate, noninvasive and safe.

Objective: To evaluate the role of magnetic resonance cholangiopancreatography (MRCP) and percutaneous transhepatic cholangiography (PTC) in the diagnosis of malignant biliary obstruction.

Patients and Methods: This study was conducted on 30 patients (18 males and 12 females) with malignant obstructive jaundice. Their ages ranged between 36 and 85 years with a mean age of 59.9 years. All the 30 examined cases in our study were subjected to MRCP and PTC to detect the diagnosis, the level of obstruction and the degree of obstruction.

Results: Both MRCP and PTC were accurate in the detection of the malignant biliary dilatation and the level of obstruction. MRCP provided adequate information in the diagnosis of the malignant lesions with sensitivity of $86.6 \%$ with low sensitivity to detect the degree of obstruction weather complete or partial $12.5 \%$ compared to PTC. MRCP detected other related malignant features in $40 \%$ of cases. PTC-related complications in 6 out of 30 cases (20\%). In 6 patients more than one puncture had to be performed during PTC to delineate the whole biliary system.

Conclusion: MRCP is accurate and non-invasive procedure for the diagnosis of the malignant obstructive jaundice and the level of biliary obstruction reserving PTC for therapeutic procedures and pre-surgical assessment of the degree of obstruction.
\end{abstract}

Keywords: Magnetic resonance, Cholangiopancreatography, Percutaneous trans-hepatic, cholangiography, Malignant obstructive jaundice.

\section{INTRODUCTION}

Malignant obstructive jaundice is a frequent clinical condition caused by malignancies of the pancreas, biliary tree, liver, or subsequent metastases in the liver or lymph nodes of the porta-hepatis ${ }^{(\mathbf{1})}$. The majority of the time, radiographic imaging is used to diagnose bile duct cancer. Imaging gives crucial information for assessing operability and the most appropriate surgical approach (2). Elevated serum enzymes may be the sole identifiable abnormality in the early stages of bile duct cancer, but after the tumour has grown large enough to cause stenosis along the biliary channel, serum bilirubin begins to rise. The major goal is to distinguish between intra- and extrahepatic causes. Imaging must next be used to establish the location and type of the obstructive lesion ${ }^{(3)}$.

MRCP is a completely non-invasive technique that provides projectional images similar to those of ERCP without administration of any contrast agents, without radiation, requires no anesthesia and less operator dependent ${ }^{(4)}$. The signal of static or slowmoving fluid-filled structures like the bile and pancreatic ducts is greatly increased by using heavily T2-weighted sequences, resulting in increased duct-tobackground contrast, so signals from the biliary system and pancreatic duct fluids are hyper-intense whereas signals from the background tissue are hypo-intense, allowing excellent description of the biliary anatomical structures ${ }^{(5)}$.

PTC studies show the level, extent and characteristics of lesions which obstruct bile ducts. Biliary obstruction is caused by many conditions, including benign and malignant diseases. Malignant biliary obstruction (MBO) is usually caused by cholangiocarcinoma, gall bladder and pancreatic malignancies, metastatic lymphadenopathy and infrequently by hepatic and advanced gastric and duodenal malignancies. Frequently the tumors are unresectable at diagnosis and only palliative treatment is possible to improve patients' quality of life ${ }^{(6)}$.

The management of obstructive jaundice is often a difficult problem for the surgeon. Many affected patients are elderly, and are poor surgical risks. Accurate information regarding the level, extent and nature of the obstructing lesion enables an early and precise decision on the type of surgical intervention, and greatly improves the progress of these patients. Tumors situated distally in the common bile duct or ampulla of Vater are often amenable to surgical resection. Detailed information on more proximal, unresectable tumors may allow the surgeon to arrange a bypass treatment for beneficial palliation, or it may 
suggest that surgery is not recommended. Inappropriate surgical examination that does not alleviate the patient's jaundice might hasten his or her death ${ }^{(7)}$.

In this study, we aimed to evaluate the role of magnetic resonance cholangiopancreatography (MRCP) and percutaneous transhepatic cholangiography (PTC) in the diagnosis of malignant biliary obstruction.

\section{PATIENTS AND METHODS}

This study was performed from January 2018 to March 2019. This study was a retrospective study and all patients' data are anonymous. It included 30 patients, 18 males and 12 females, whose ages ranged between 36 and 85 years (mean age: 59.9 years). They were referred to the Radiology Department for MRCP and PTC examinations of the biliary tract. The patients presented mainly with jaundice and one or more of the following signs and symptoms: biliary colic, fever and rapid loss of weight. MRCP, PTC and complementary ultrasound were done for all 30 cases. MDCT was done for 20 cases. Biopsy was done for 25 cases.

Laboratory investigations included the evaluation of total and direct bilirubin, serum liver enzymes, and tumor markers for all cases. The final diagnosis was reached using histo-pathologic data and follow up imaging.

Inclusion criteria: Patients with malignant biliary obstruction, prothrombin concentration $>60 \%$ and platelet count $>50,000$ platelet $/ \mathrm{ml}$.

Exclusion criteria: Patients with benign biliary obstruction, patients with serious coagulation disorder (prothrombin concentration $<60 \%$ or platelet count $<$ $50,000 / \mathrm{ml}$ ), renal impairment and ascites as a relative contraindications.

\section{Imaging: \\ MRCP technique \\ Patient preparation}

Patients were instructed to fast for at least 6 hours before MRCP examination. Asking about full history and history of cardiac pacemakers and any ferromagnetic materials in a critical location that degrade the image and are potentially hazardous to the patients. No pre-medications or contrast medium were administrated.

\section{Technique}

The 30 examined patients were subjected to MRCP examination, which was performed on MRI machine (GE HEALTH CARE 1.5-T MRI scanner, USA) with a circular surface (synergy body) coil using breathhold 2D single-slice FSE and non-breath- hold multislice acquisition followed by 3D MIP reconstruction with complementary axial or coronal T2 weighted MR images. 2D MRCP using single slice acquisition was performed using the following parameters shown in table (1).

Table (1): The parameters used for MRCP

\begin{tabular}{|c|c|c|c|c|c|c|}
\hline & $\begin{array}{c}\text { Axial } \\
\text { heavy } \\
\text { T2 }\end{array}$ & $\begin{array}{c}\text { Axial T2 } \\
\text { propeller } \\
\text { fat sat }\end{array}$ & $\begin{array}{l}\text { Coronal } \\
\text { 3D thin } \\
\text { cuts }\end{array}$ & $\begin{array}{l}\text { Axial } \\
\text { 3D thin } \\
\text { cuts }\end{array}$ & \begin{tabular}{|c|} 
Coronal \\
$2 \mathrm{D}$
\end{tabular} & $\begin{array}{c}\text { Axial } \\
\text { diffusion }\end{array}$ \\
\hline $\begin{array}{l}\text { Patient } \\
\text { position }\end{array}$ & Supine & Supine & Supine & Supine & Supine & Supine \\
\hline FOV & 38 & 38 & 36 & 32 & 36 & 38 \\
\hline \begin{tabular}{|c|}
$\begin{array}{c}\text { Slice } \\
\text { number }\end{array}$ \\
\end{tabular} & 30 & 30 & 100 & 84 & 30 & 25 \\
\hline $\begin{array}{l}\text { Slice } \\
\text { thick- } \\
\text { ness } \\
\end{array}$ & $6 \mathrm{~mm}$ & $6 \mathrm{~mm}$ & $1 \mathrm{~mm}$ & $2 \mathrm{~mm}$ & $5 \mathrm{~mm}$ & $\begin{array}{r}5 \\
\mathrm{~mm}\end{array}$ \\
\hline TR & $\begin{array}{c}14000 \\
\mathrm{msec}\end{array}$ & $\begin{array}{l}2000 \\
\text { msec }\end{array}$ & $\begin{array}{l}2000 \\
\text { msec }\end{array}$ & $\begin{array}{l}3000 \\
\text { msec }\end{array}$ & $\begin{array}{l}2727 \\
\text { msec }\end{array}$ & $\begin{array}{l}8700 \\
\text { msec }\end{array}$ \\
\hline $\mathrm{TE}$ & $\begin{array}{l}300 \mathrm{~m} \\
\mathrm{sec}\end{array}$ & $\begin{array}{r}95 \\
\text { msec }\end{array}$ & $\begin{array}{r}529 \\
\text { msec }\end{array}$ & $\begin{array}{r}463 \\
\mathrm{msec}\end{array}$ & $\begin{array}{r}102 \\
\text { msec }\end{array}$ & $\begin{array}{r}57 \\
\mathrm{msec}\end{array}$ \\
\hline $\begin{array}{l}\text { Scan } \\
\text { time: }\end{array}$ & $\begin{array}{l}1: 30 \\
\min \end{array}$ & $\begin{array}{r}5 \\
\min \end{array}$ & $\begin{array}{c}4 \\
\min \end{array}$ & $\begin{array}{l}2: 35 \\
\min \end{array}$ & $\begin{array}{l}2: 44 \\
\min \end{array}$ & $\begin{array}{l}1: 27 \\
\min \end{array}$ \\
\hline \begin{tabular}{|c|} 
Matrix \\
size:
\end{tabular} & $\begin{array}{l}256 \times 1 \\
92\end{array}$ & $\begin{array}{l}320 x \\
320\end{array}$ & $\begin{array}{l}288 x \\
288\end{array}$ & $\begin{array}{l}224 x \\
224\end{array}$ & $\begin{array}{l}320 \times 3 \\
20\end{array}$ & $\begin{array}{c}92 \mathrm{x} \\
128\end{array}$ \\
\hline NEX & 1 & 1.5 & 1 & 1 & 1 & 1 \\
\hline
\end{tabular}

The angle of the coronal image could be changed several times to obtain satisfactory results.

Axial T2-weighted MR images of the upper abdomen was performed first before MRCP as they considered as a guide to determine the correct obliquity of the coronal oblique sections of MRCP images. The angle of MRCP examination was chosen from the section at which the biliary lesion appeared.

\section{PTC technique: \\ Patient preparation:}

Before imaging, the method of PTC was fully explained to all the patients, any coagulation imbalance was corrected before the intervention by administration of vitamin K, platelets and fresh frozen plasma. Patients were instructed to fast for 6-8 hours before the procedure and written informed consent was signed by all patients.

\section{Technique:}

The 30 examined patients were subjected to PTC examination, which was performed using Fluoroscopic x-ray unit: Philips Allura clarity angiography unit and Toshiba - Infinix, Ultrasound devices: Toshiba-Nemio XG, GE LOGIQ P6 and Toshiba-Xario,2000, 18 G Chiba needle, 6Fr Introducer sheath, $0.035^{\prime}$ super-lubricity (Hydrophilic) angled tip guide wire and 5 Fr Cobra Catheter. The procedure was performed under sterile conditions, with the patient in supine position. The procedure was performed using percutaneous local anesthesia (10 $\mathrm{ml}$ of $2 \%$ lidocaine) and under conscious sedation. Right-sided approach was preferred in most cases, reserving the left-side approach to cases where there was a small amount of 
ascites or colon interposition that would not permit a right-side puncture.

A peripherally dilated bile duct was punctured with $18 \mathrm{G}$ Chiba needle, under US for the initial ductal puncture and fluoroscopic guidance, which were used to guide wire and catheter insertion, manipulation, and advancement. Entry into the bile duct was recognized by injection of diluted contrast material (CM) through the Chiba needle. CM injected into the bile duct flows slowly away like wax flowing down a candle. 0.035" Terumo hydrophilic guide wire was inserted through the Chiba needle, and negotiated centrally towards the porta-hepatis. Introducer sheath was placed over the guide wire then $\mathrm{CM}$ was injected from the side port of the sheath, accurately delineating the location, length, morphology of the biliary obstruction and the patency of the cystic duct through postero-anterior, right and left posterior oblique and caudo-cranial and craniocaudal positions.

\section{Ethical consent:}

An approval of the study was obtained from Menoufia University Academic and Ethical Committee. Every patient signed an informed written consent for acceptance of the examination.

\section{Statistical analysis:}

The collected data were coded, tabulated, and statistically analyzed using IBM SPSS statistics
(Statistical Package for the Social Sciences) software version 22.0, IBM. Descriptive statistics were presented for quantitative data as mean $\pm \mathrm{SD}$ (standard deviation) and were compared by independent t-test, while for qualitative data it was presented as number and percentage and were compared by Chi-square $\left(\mathrm{X}^{2}\right)$ test. $\mathrm{P}$ value less than 0.05 was considered statistically significant and less than 0.001 was considered highly significant.

\section{RESULTS}

All the patients in the study were presenting by jaundice separately or in conjunction with other signs just as biliary colic, fever and progressive loss of weight. Elevated bilirubin level both total and direct bilirubin were seen at all the cases. Total bilirubin levels are ranging from 3.6 to 43.5. Direct bilirubin levels are ranging from 1.9 to 30.6 . The findings in the biliary tree of the 30 patients showed findings of different pathologic entities.

Cholangiocarcinoma included the largest number of patients $(46.7 \%)$, while $26.7 \%$ presented with pancreatic head carcinoma, $10 \%$ presented with periampullary carcinoma, $10 \%$ presented with hepatocellular carcinoma causing biliary obstruction, $3.3 \%$ presented with gall bladder carcinoma and $3.3 \%$ presented with gastric carcinoma (Fig. 1).

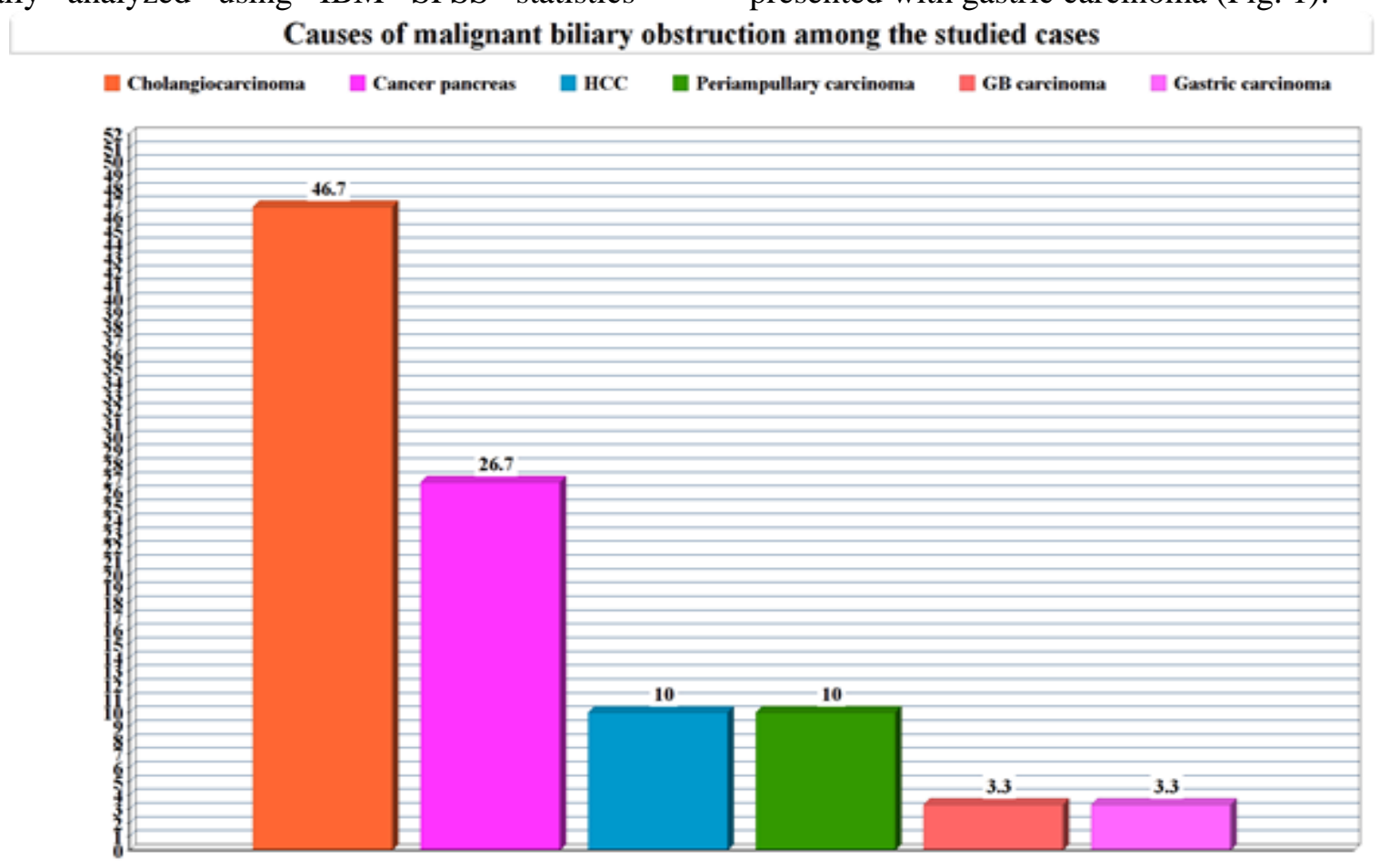

Figure (1): Causes of obstruction among the studied cases.

Final diagnosis was reached by correlating with histopathological, laboratory data and follow up imaging. There was perfect symmetry between the results for the diagnosis of malignant biliary obstruction in relation with the results of various confirmatory modalities.

Hilar malignant biliary obstruction was more common than distal type about 19 cases out of 30 cases $(63.3 \%)$. According to Bismuth classification of the hilar biliary obstruction, the most common Bismuth type among the studied cases was Bismuth IV, 10 cases of the studied 30 cases (33.3\%) (Fig. 2). 


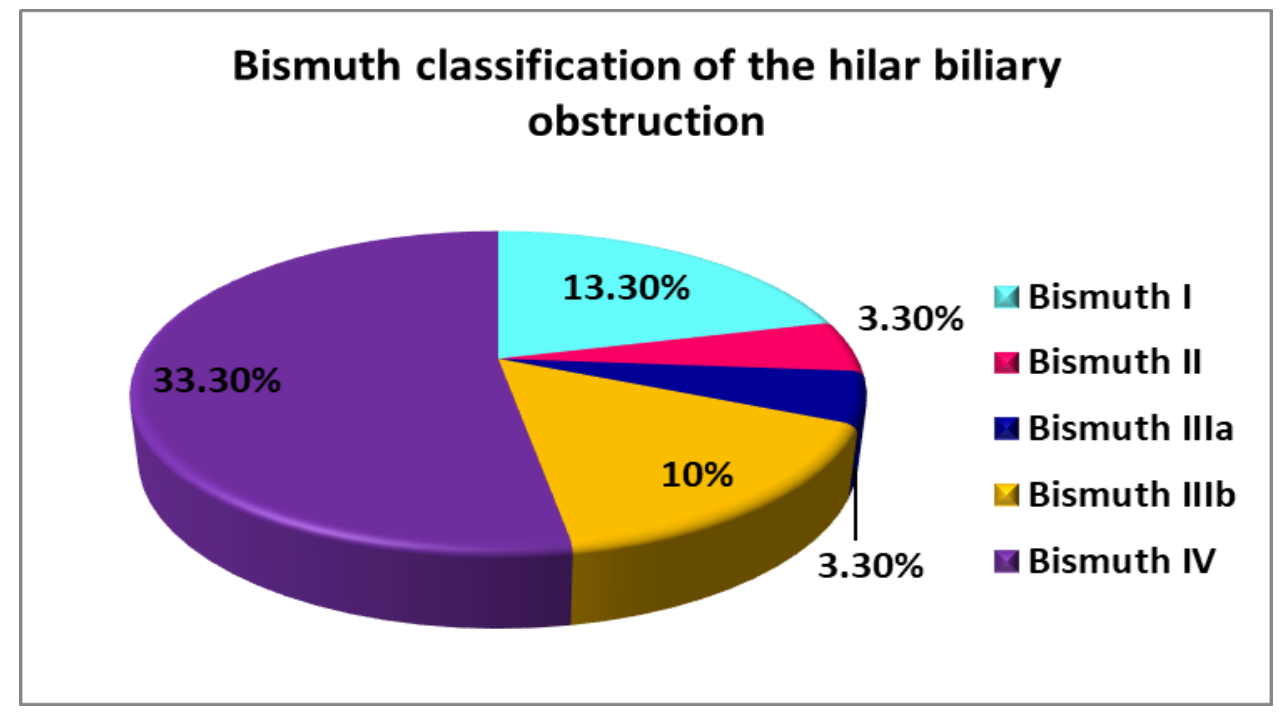

Figure (2): Bismuth classification of the hilar biliary obstruction.

Both MRCP and PTC could accurately detect the level of obstruction in all 30 cases, but MRCP was superior in the detection of the malignant cause of obstruction with sensitivity of $86.6 \%$ but it showed low sensitivity to detect the degree of obstruction whether complete or partial (12.5\%) compared to PTC. PTC detected 8 cases from 30 cases (26.7\%) with incomplete biliary obstruction, while MRCP detected one case from 30 cases with incomplete biliary obstruction (3.3\%) (Fig. 3).

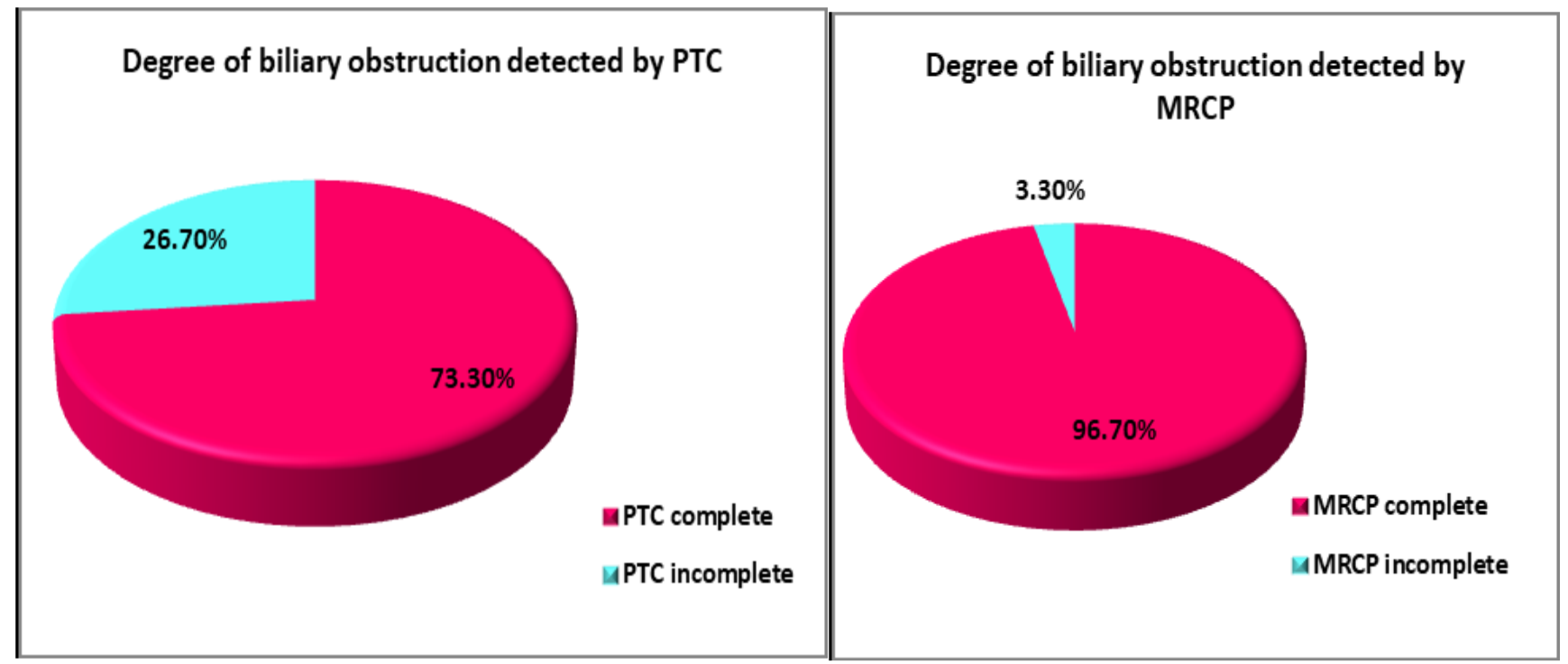

Figure (3): The detectability of the degree of obstruction among the studied groups by MRCP and by PTC.

MRCP detected other related malignant features in 12 out of 30 cases (40\%). Five cases (16.6\%) with regional lymph nodal deposits, four cases (13.3\%) with portal vein invasion, two cases $(6.6 \%)$ with hepatic deposits and one case (3.3\%) with peritoneal deposits. Post-PTC complications were found at 6 out of 30 cases $(20 \%)$. Two cases $(6.6 \%)$ complicated with Infection at puncture site, one case (3.3\%) complicated with one of biliary venous fistula, biliary leakage, renal impairment or vagal attack respectively.

Among our studied cases in 24 out of 30 cases (80\%) one puncture was needed during PTC to image the whole biliary system and in 6 out of 30 cases two punctures were needed to image the whole biliary system. 


\section{CASE 1}

53 years old female patient presented by abdominal pain and gradual progressive jaundice.

(A) Axial CT with contrast: Polypoidal enhancing mural thickening at the gastric pyloric region measuring $2 \mathrm{~cm}$ (Red arrow).

(B) MRCP findings: (B) Coronal 3D with MIP: There was moderate dilatation of the intrahepatic biliary radicles, right and left hepatic ducts and proximal common hepatic ducts (Bismuth I) (Red arrow). (C) Axial T2WI: There was moderate dilatation of the intrahepatic biliary radicals (Red arrow).

PTC findings: Cranio-caudal view: There was moderate dilatation of the intrahepatic biliary radicles (Red arrow) and contrast passage through the incomplete hilar obstruction (Yellow arrow). Postero-anterior view: There was moderate dilatation of intra-hepatic biliary radicles. The case was gastric carcinoma.

Figure (4) Case 1:
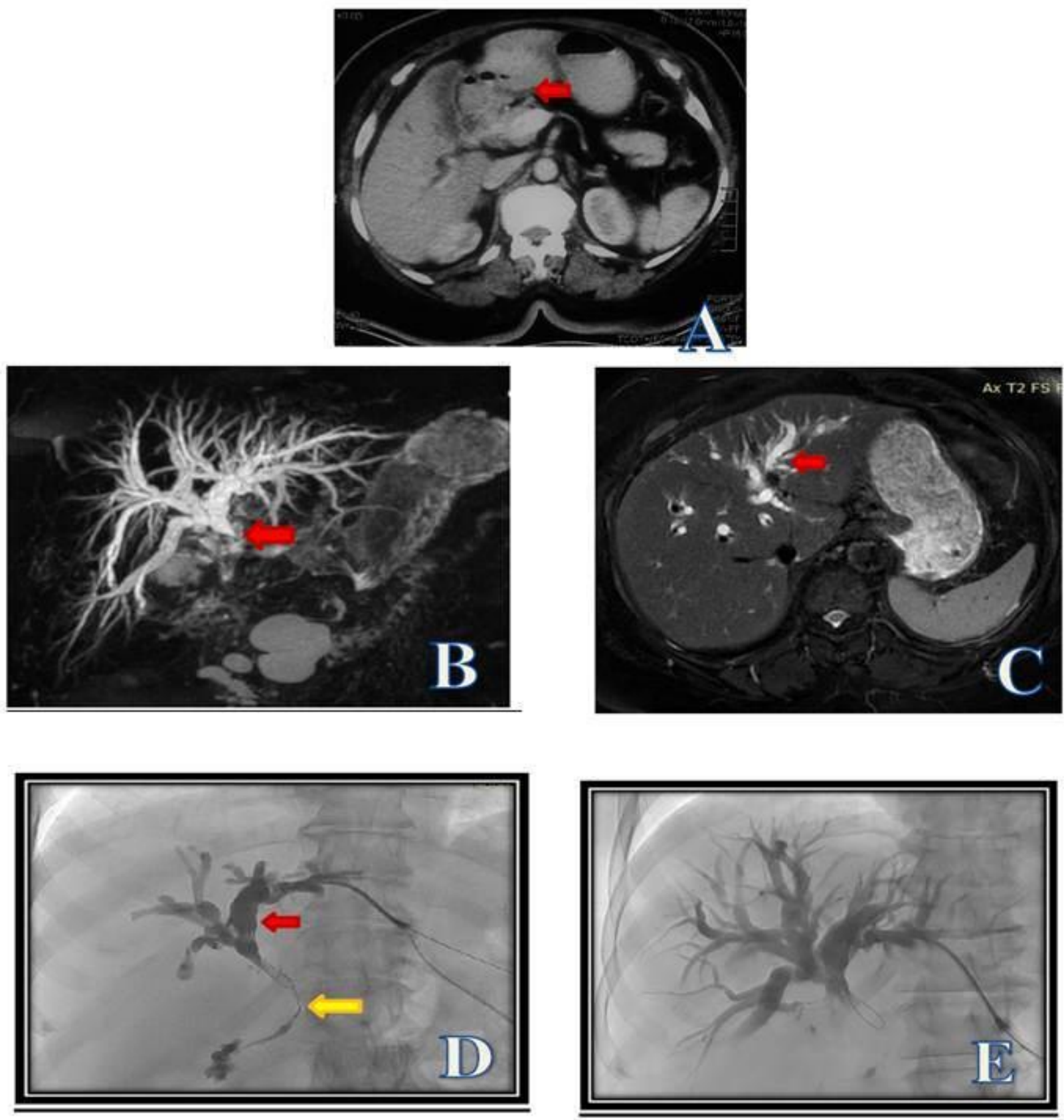


\section{CASE 2:}

61 years old male patient with hepatomegaly presented by progressive jaundice and loss of weight.

Triphasic CT finding: Enhancing focal lesion at the confluence of the hepatic ducts measuring about $2 \times 2 \mathrm{~cm}$ (figure A red arrow) with moderate bilobar intra-hepatic biliary radicles (figure B yellow arrow). Another heterogeneously enhancing hepatic focal lesion at segment VIII/IV (figure B red arrow).

MRCP findings: (C \& D) Axial T2 FS WIs: Focal lesion with high signal at T2 (figure $\mathrm{C}$ red arrow) at the confluence of hepatic ducts compressing right and left ducts with moderate bilobar biliary radicles and extension to the right hepatic duct (Bismuth IIIa) (figure D red arrow). (E) Coronal 3D MRCP with MIP: hilar compression of both hepatic ducts (red arrow) with moderate dilated bilobar biliary radicles (yellow arrow).

PTC findings: (F) Cranio-caudal: Moderate bilobar biliary radicles dilatation (red arrow). (G) Left posteror oblique): Moderate bilobar biliary radicles dilatation with filling defect at the confluence of hepatic ducts extending to right hepatic duct (red arrow) with incomplete obstruction of the left biliary system (yellow arrow). The case was cholangiocarcinoma.

Figure (5): Case 2:
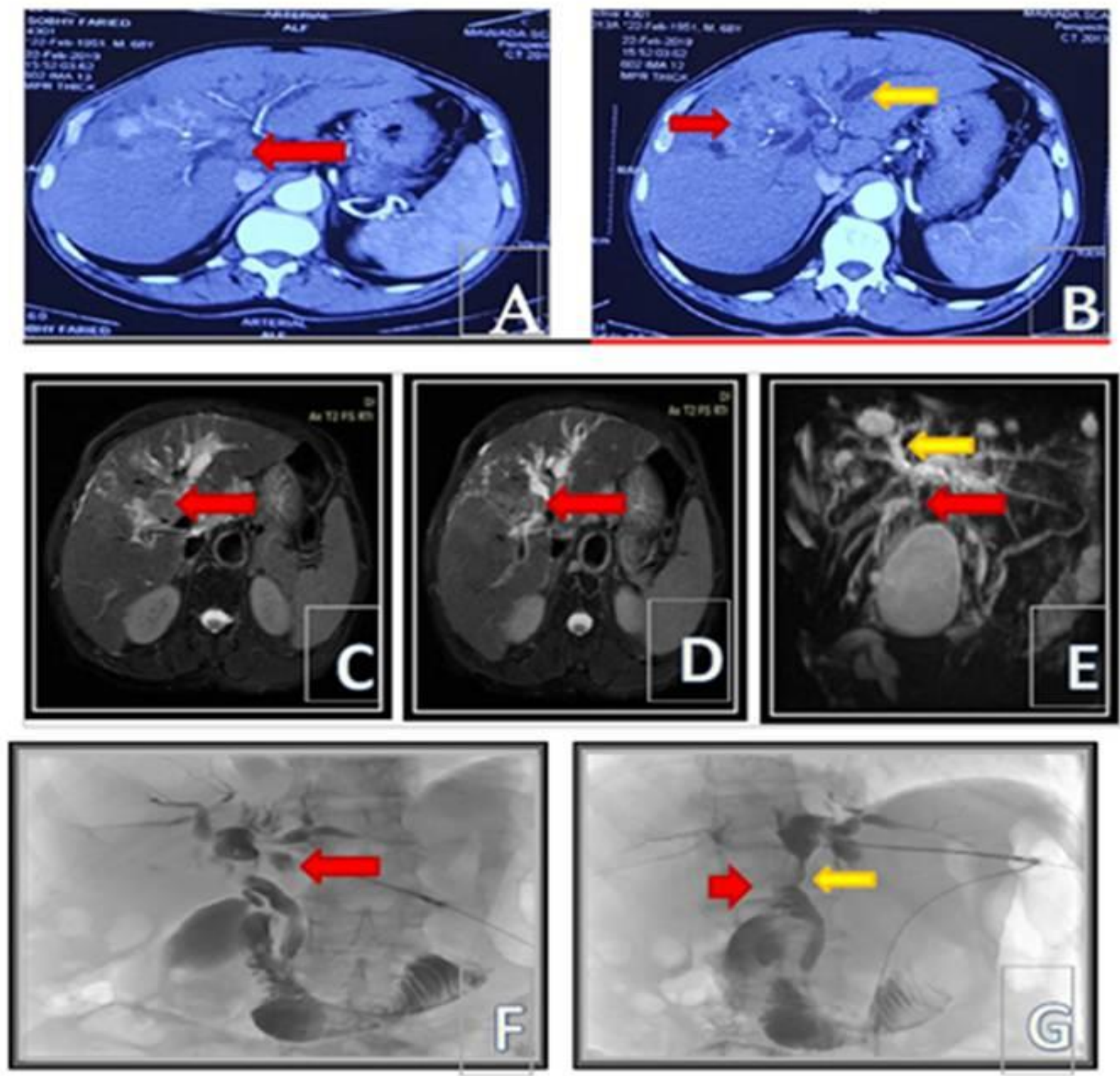


\section{CASE 3:}

63 years old female patient presented by abdominal pain, fever and gradual progressive jaundice.

US findings: There was dilated IHBR, dilated CBD with soft tissue seen at its distal end (red arrow). Hypoechoic hepatic focal lesion was seen (yellow arrow).

MRCP findings: (B) Coronal T2 FS: There was dilated CBD (red arrow), IHBRD and calcular gall bladder (yellow arrow). (C) Coronal 3D with MIP: There was dilated CBD and dilated IHBR (yellow arrow) with filling defect measuring $2.3 \mathrm{~cm}$ at its distal end $2.8 \mathrm{~cm}$ from the papillae (yellow arrow). (D) Axial Heavy T2WI: There was dilated CBD and hepatic segment VI high signal focal lesion (red arrow).

PTC findings: (E) Postero-anterior view and (F) Left posterior-anterior view: There was moderate dilatation of IHBR (blue arrow) and dilated CBD (yellow arrow) with filling defect measuring $2.3 \mathrm{~cm}$ at its distal end $3.7 \mathrm{~cm}$ from the papillae (red arrow). The case was distal cholangiocarcinoma.

Figure (6): Case 3:
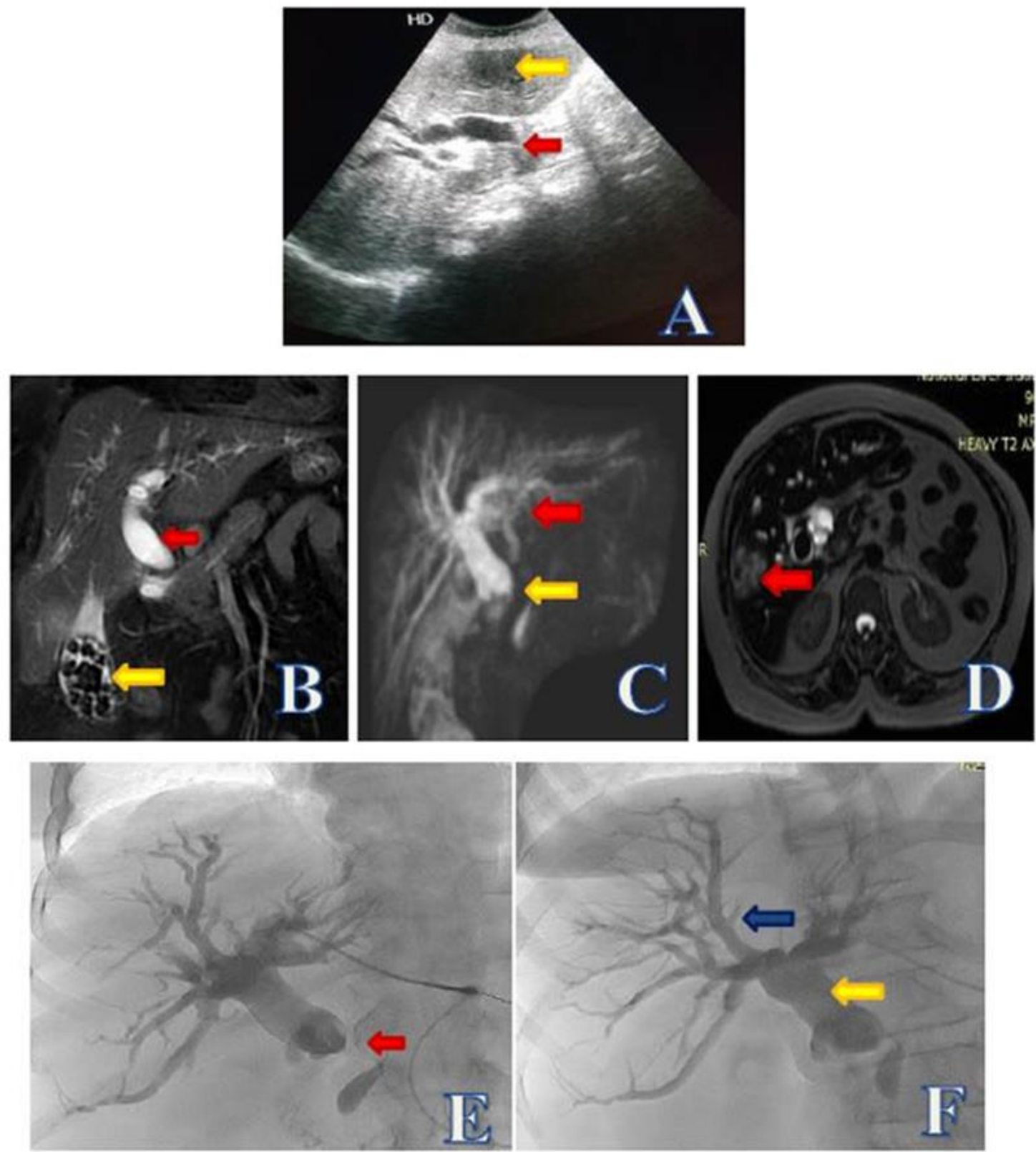


\section{CASE 4:}

58 years old male patient with gradual progressive jaundice and abdominal pain.

CT findings: (A) \& (B) axial and coronal CT with contrast: Situs inversus totalis. Hilar hypodense soft tissue mass lesion (figure B red arrow) that reflects mild bilobar intra-hepatic biliary radicles dilatation (figure A yellow arrow). MRCP findings: (C)) Axial Heavy T2WI, (D) Coronal T2WI \& (E) Axial 3D thin cuts: Situs inversus totalis. Blue arrow refers to shrunken left lobe with mildly dilated intra-hepatic biliary radicles, red and yellow arrows refer to hypo-intense hilar focal lesion.

PTC findings: $(F \& G)$ Postero-anterior view: There is mildly dilated intra-hepatic biliary system (yellow arrow) with hilar filling defect (red arrows). Average CBD, cystic and pancreatic ducts. The case was cholangiocarcinoma.

Figure (7): Case 4:
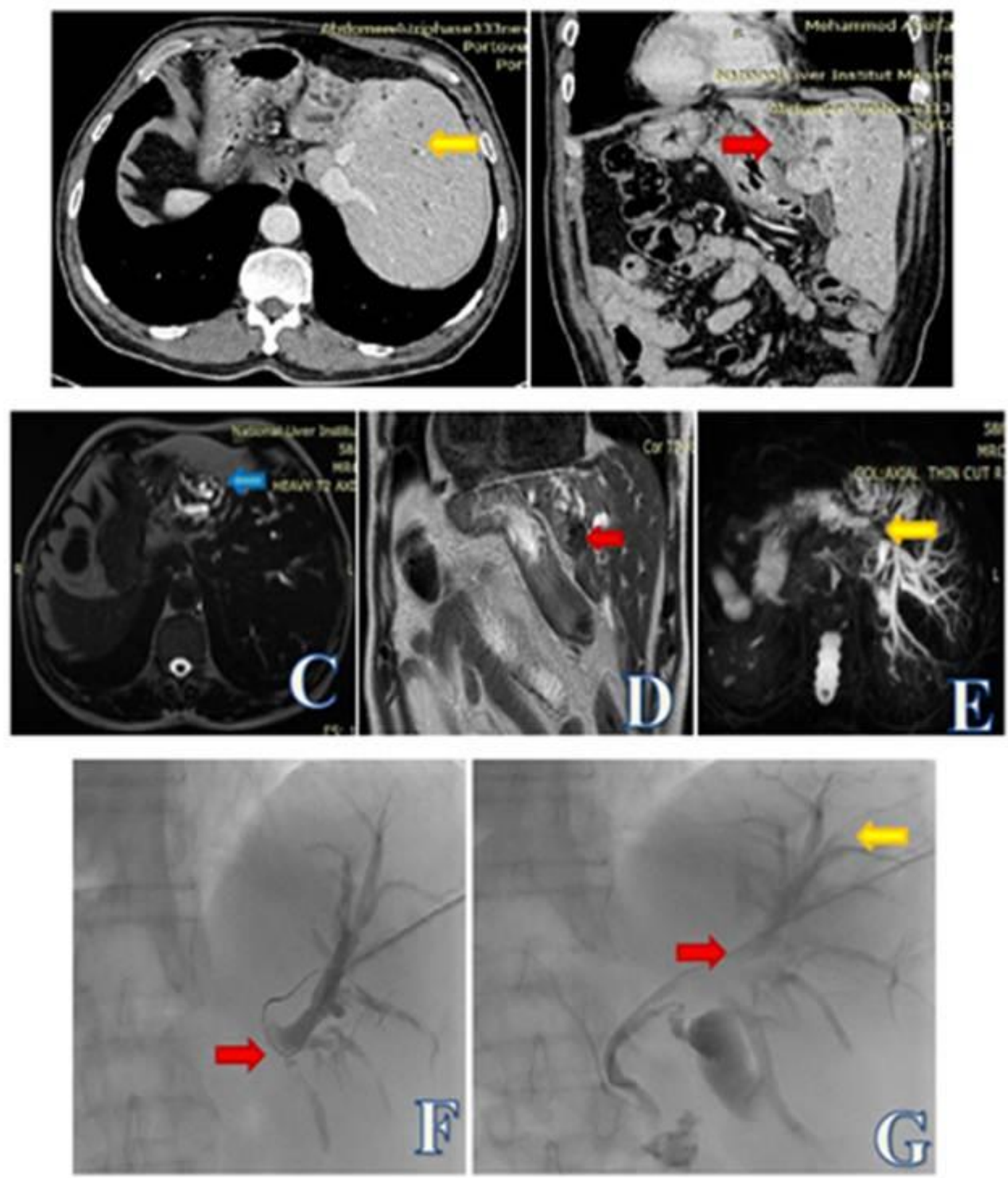


\section{CASE 5:}

40 years old female patient presented by abdominal pain, loss of weight and gradual progressive jaundice. CT findings: (A) \& (B) axial CT with contrast: There is hepatic focal lesion with peripheral enhancement (red arrow) and dilated IHBRD (yellow arrow).

MRCP findings: (C) Axial T2 FS: There was hepatic focal lesion with high signal intensity (red arrow) and dilated left IHBRD (yellow arrow) and right segmental IHBRD (blue arrow). (D) Axial Heavy T2WI: There was left mild IHBRD (yellow arrow) and right segmental IHBRD (blue arrow). (E) Coronal 3D with MIP: There was hilar obstruction (Bismuth IV) (blue arrow).

PTC findings: (F) Postero-anterior view and $(\mathrm{G})$ Caudo-cranial view: There was mild dilatation of IHBR (red arrows) with hilar filling defect (blue arrow). Displaced calcular gall bladder (yellow arrow). The case was cholangiocarcinoma

Figure (8): Case 5:
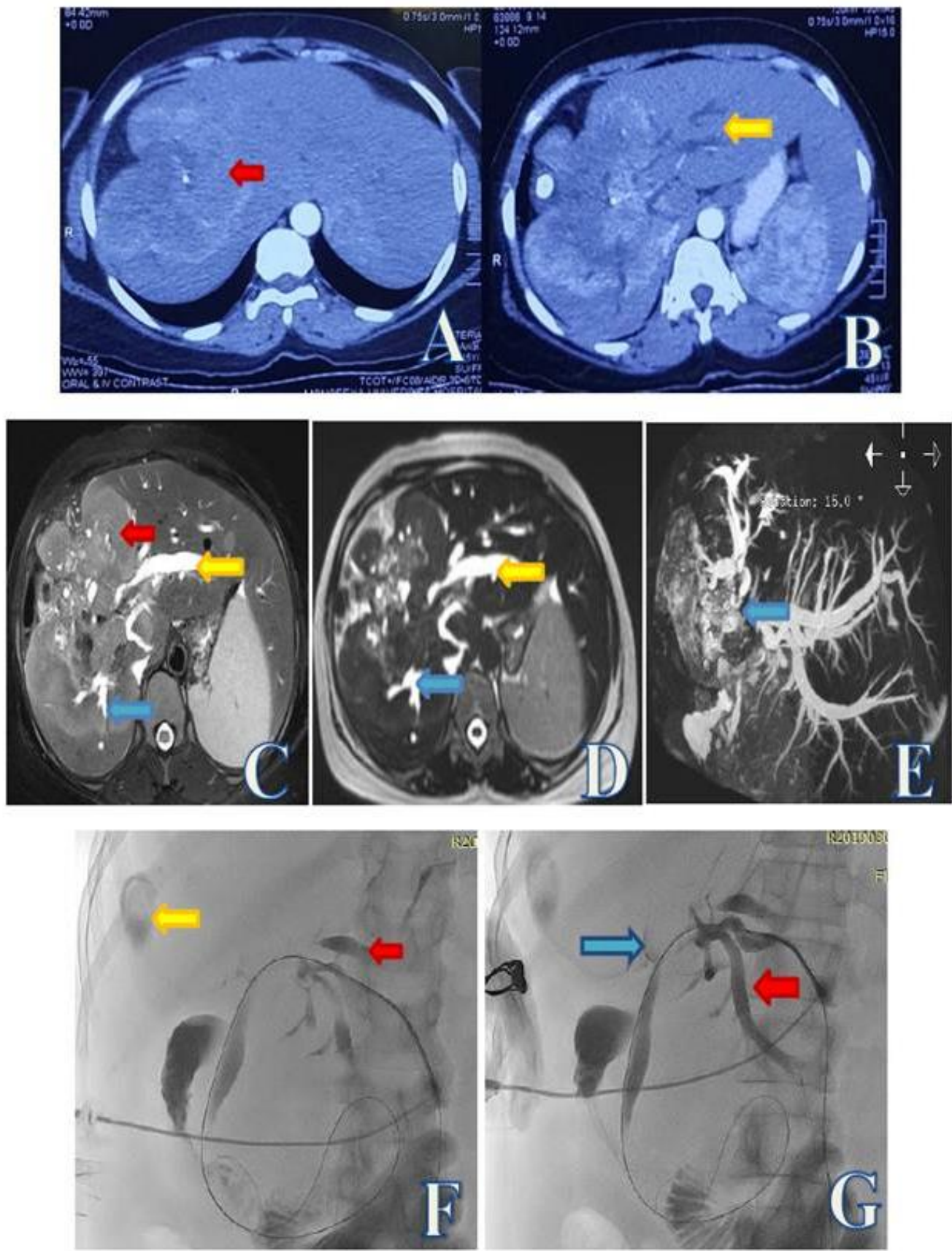


\section{DISCUSSION}

The study group consisted of 30 patients (18 males and 12 female). Their ages ranged between 36 and 85 years, with a mean age of 59.9 years. Biochemical criteria of the patients revealed elevation of both total and direct bilirubin in all cases, which is in agreement with a study done by Madhok et al. ${ }^{(8)}$ that revealed elevation of serum bilirubin level \& ALP in all patients with malignant obstructive jaundice.

In our study cholangiocarcinoma included the largest number of patients 14 out of 30 cases $(46.7 \%)$, while eight cases out of 30 cases $(26.7 \%)$ presented with pancreatic head carcinoma. This is contradictory to Zhong et al. ${ }^{(9)}$ where they reported that pancreatic head carcinoma was the commonest cause of malignant obstructive jaundice, 11 out of 34 cases $(32 \%)$, while cholangiocarcinoma was the second cause 9 out of 34 cases (26\%).

Our study showed that both MRCP and PTC with $100 \%$ accuracy in detecting the level of obstruction. Singh et al. (4) study showed 100\% sensitivity, specificity and diagnostic accuracy of $\mathrm{MRCP}$ in detecting periampullary carcinoma. MRCP showed significant preference in the ability to detect the cause of malignant obstruction with sensitivity of $86.6 \%$ while PTC could only detect the level of obstruction. Our study is in agreement with the study of Shukla et al. ${ }^{\left({ }^{(10)}\right.}$ who showed that MRCP was able to detect the level of obstruction in $95.45 \%$ and the cause in $87.50 \%$ of cases.

In our study, obstruction was hilar in 19 out of 30 cases $(63.33 \%)$ and was distal in 11 cases (36.66 $\%)$. While, Zhong et al. ${ }^{\left({ }^{(9)}\right.}$ reported that among the 82 patients with pancreato-biliary diseases, 8, 60 and 14 cases had pancreato-biliary obstructive locations in intra-hepatic, extra-hepatic bile duct and in main pancreatic duct, respectively.

In our study, cases with hilar obstruction were classified according to Bismuth classification into I, II, IIIa, IIIb and IV. The incidence of Bismuth type IV was the most common Bismuth type, 10 cases of the studied 30 cases $(33.3 \%)$. In Hennedige et al. (11) study, cholangiocarcinoma (CCA) cases are classified based on their anatomic location as intrahepatic CCA (iCCA), perihilar CCA (PCCA) and distal (DCCA) subtypes and extr-ahepatic cholangiocarcinoma (90\%) was more common than intra-hepatic cholangiocarcinoma (10\%).

Our study showed superiority of PTC in detection of the degree of the obstruction whether it was complete or incomplete. PTC detected 8 out of 30 cases with incomplete biliary obstruction $(26.7 \%)$, while MRCP detected one case with incomplete biliary obstruction (3.3\%) with low sensitivity (12.5\%) and $100 \%$ specificity. In Legge et al. ${ }^{(12)}$ study, PTC performed on 13 cases, one case $(7.6 \%)$ was with partial occlusion of the common bile duct.
MRCP detected other related malignant features in $40 \%$ of cases, $16.6 \%$ with regional lymph nodal deposits, $13.3 \%$ with portal vein invasion, $6.6 \%$ with hepatic deposits and $3.3 \%$ with peritoneal deposits, while Madhok et al. ${ }^{(8)}$ reported other malignant features in 50\% of cases ( lymph nodal deposits).

PTC is an invasive technique that assesses biliary ducts and has the added advantage of further interventional drainage. Our study revealed that no complications of PTC at $80 \%$ of cases and complications occurred at 6 cases $(20 \%)$. Two cases (6.6\%) complicated with infection at the puncture site, one case $(3.3 \%)$ complicated with one of biliary venous fistula, biliary leakage, renal impairment or vagal attack. The study performed by Chaudhary $\boldsymbol{e t}$ al. (13) on 26 patients showed that, three patients (11.5\%) developed cholangitis after PTC and four patients (15.4\%) needed analgesics for pain relief.

PTC demonstrated the entire biliary tract from a single puncture in 24 out of 30 cases $(80 \%)$. In the remaining 6 patients $(20 \%)$ more than one puncture was necessary to image the whole biliary system. Chaudhary et al. ${ }^{(13)}$ study on 26 patients showed that, in 18 patients $(69.3 \%)$ PTC demonstrated the entire biliary tract from a single puncture of the right-sided duct system. In the remaining eight patients $(30.7 \%)$ more than one puncture was necessary to delineate the biliary anatomy.

Finally, both MRCP and PTC have a great role in the diagnosis of malignant biliary obstruction. MRCP is a non-invasive technique that provides projectional images without administration of contrast agents, without radiation, requires no medications, less operator dependent and no reported complications. MRCP can detect the nature of the obstructing lesion, the level obstruction and the other surrounding malignant features.

PTC has several advantages in the diagnosis of malignant from benign biliary obstruction, the level of obstruction, the pre-surgical assessment of the degree of obstruction whether complete or partial and it also offers potential therapeutic options. However, PTC is invasive technique and associated with an unacceptable rate of procedure-related complications.

\section{CONCLUSION}

This study sheds light on the great role of MRCP and PTC in the diagnosis of malignant obstructive jaundice. MRCP is non-invasive technique, which is highly accurate in the detection of the malignant lesions, the level of the biliary obstruction and delineating the related malignant sequences. PTC played a good role in the detection of the level of the biliary obstruction, diagnosis of malignant from benign biliary obstruction, diagnosis of the degree of obstruction and it also offers potential therapeutic options. 
We recommend that MRCP should be the imaging modality of choice for diagnosis of malignant obstructive jaundice, reserving PTC for therapeutic procedures and pre-surgical assessment of the degree of obstruction.

\section{REFERENCES}

1. Motohara T, Semelka R, Bader T (2003): MR cholangiopancreaticography. Radiol Clin North Am., 41 (1): 89-96.

2. Madhusudhan $\mathrm{K}$, Gamanagatti $\mathrm{S}$, Srivastava $\mathrm{D}$ et al. (2016): Radiological interventions in malignant biliary obstruction. World Journal of Radiology, 8 (5): 518-23.

3. Flucher A, Turner $M$ (2002): $\quad M R$ cholangiopancreaticography. Radiol Clin North Am., 40 (6): 1363-1376.

4. Singh A, Mann H, Thucral C et al. (2014): Diagnostic Accuracy of MRCP as Compared to Ultrasound/CT in Patients with Obstructive Jaundice. Journal of Clinical and Diagnostic Research, 14: 103-107.

5. Soto J, Lopera J, Munera F et al. (2000): Biliary obstruction: findings at MR cholangiography and cross sectional MR imaging. Radiographics, 20: 353-366.

6. Katabathina V, Dasyam A, Hosseinzadeh $\mathrm{K}$ et al. (2015): Adult Bile Duct Strictures: Role of MR Imaging and MR Cholangiopancreatography in Characterization. Radiographics, 34 (3): 565-86.
7. Liang C, Mao H, Wang Q et al. (2011): Diagnostic Performance of Magnetic Resonance Cholangiopancreatography in Malignant Obstructive Jaundice. Cell Biochemistry and Biophysics, 61 (2): 383-388.

8. Madhok R, Rastogi S (2015): Role of 3.0 Tesla Magnetic Resonance Cholangiopancreatography in Obstructive Jaundice with Cyto/Histopathological or Surgical Correlation. Int J Sci Stud., 3: 1-7.

9. Zhong L, Yao Q, Li L et al. (2003): Imaging diagnosis of pancreatobiliary diseases: a control study. World Journal of Gastroenterology, 9 (12): 2824-2827.

10. Shukla V, Upadhyaya V, Upadhyaya D et al. (2009): Comparative assessment of imaging modalities in biliary obstruction. Indian Journal of Radiology and Imaging, 16 (4): 577.

11. Hennedige T, Neo W, Venkatesh $S$ (2014): Imaging of malignancies of the biliary tract- an update. Cancer Imaging, 14: 14-26.

12. Legge D, Hennessy T (1999): Percutaneous transhepatic cholangiography in the management of obstructive jaundice. David A. Legge and Thomas Hennessy Departments of Radiology and Surgery, St. Finbarr's Hospital, Cork., Pp: 30$43 . \quad$ https://www.radiology.ie/images/uploads /2012/01/Combined-Annual-Reports.pdf

13. Chaudhary A, Negi S, Puri S et al. (2002): Comparison of magnetic resonance cholangiography and percutaneous transhepatic cholangiography in the evaluation of bile duct strictures after cholecystectomy. The British Journal of Surgery, 89 (4): 433-436. 